\title{
Titel/Title: Die National Mall in Washington D.C. - Einheit und Differenz des demokratischen Souveräns
}

Autor*innen/Author(s): Philip Manow

Veröffentlichungsversion/Published version: Postprint

Zeitschriftenartikel/Journal article

\section{Empfohlene Zitierung/Recommended citation:}

Manow, Philip (2018): Die National Mall in Washington D.C. - Einheit und Differenz des demokratischen Souveräns. In: Leviathan, Sonderband, 34, 182-195.

Verfügbar unter/Available at:

(wenn vorhanden, bitte den DOI angeben/please provide the DOI if available)

doi.org/10.5771/9783845285405-181

Zusätzliche Informationen/Additional information:

Der Autor kann kontaktiert werden unter:

manow@uni-bremen.de 
Philip Manow ${ }^{1}$

Die National Mall in Washington D.C. - Einheit und Differenz des demokratischen Souveräns

\section{Einleitung}

Wir meinen zu wissen, wofür der Barockgarten steht: geometrische Raumbeherrschung ohne Raumbegrenzung, d.h. ins Unendliche ausgreifender Herrschaftsanspruch; panoptischer Herrscherblick entlang langer Blickachsen; der absolutistische Fürst als Sonne, deren Strahlen » ungehindert die Tiefe des Gartenraums im Sinne des coup d'œil erhellen«, so Horst Bredekamps Charakterisierung unseres gängigen Bildes. ${ }^{2}$ Kurz: wir verstehen den Barockgarten als exemplarischen Aus- druck des Ancien Régime. Doch die nähere Betrachtung zeigt, wie so häufig, dass ungefähr das Gegenteil zutrifft. Der Barockgarten, beispielsweise der Versailler Schlossgarten, wird bereits frühzeitig öffentlich zugänglich gemacht (in Versailles vor 1718). In ihm sind die höfisch-formalen Umgangsformen suspendiert, d.h. er bietet Raum für ungezwungene Begegnungen über Standesgrenzen hinweg. Er entwickelt sich schließlich zum »Reflexionsraum einer weitgefassten Öffentlichkeit «. ${ }^{3} \mathrm{Er}$ ist also weniger absolutistischer Herrschaftsraum als vielmehr liminal space zwischen protobürgerlicher Gesellschaft und adeligem Hof. Zugleich zeigt sich sein vorgeblich liberales Gegenstück, der englische Landschaftsgarten, bei genauerer Betrachtung als ausgesprochen ambivalentes Gebilde, auf umfassenden enclosures, also Zwangsenteignungen, beruhend, und in seiner inszenierten Grenzenlosigkeit Komplement einer bellizistischen Empire-Ideologie, die die Freiheit weltweiter Handelswege mit Gewalt durchzusetzen gewillt war.

Für die National Mall in Washington D.C. werden diese Beobachtungen bedeutsam, sobald man in Rechnung stellt, dass deren Konzeption auf den französischen Architekten Pierre L'Enfant zurückgeht, der von George Washington mit der Hauptstadtplanung beauftragt worden war, wobei L'Enfants Planungen wesentlich vom Versailler Schlosspark inspiriert wurden. ${ }^{4}$ Ein Barockgarten im Zentrum der neuen Welthauptstadt der demokratischen Revolution - wie geht das zusammen? Die übliche Reaktion ist dann zunächst auch nur von Ratlosigkeit geprägt: $(\leftarrow$ S. 182)

»Wenn irgend etwas, dann hätte wohl diese totale Umgestaltung der politischen Verhältnisse zu einer Wandlung der barocken Ordnung führen müssen. [...] Was aber sehen wir? [...] Obwohl L'Enfant ein überzeugter Republikaner war, entsprach sein Entwurf der neuen Hauptstadt in jeder Hinsicht dem, was sich die Architekten und Diener des Despotismus ursprünglich vorgestellt hatten ${ }^{5}{ }^{5}$

Was vor dem Hintergrund des hergebrachten Barockbilds als reines Rätsel er- scheinen muss, wird schließlich mit dem eher reflexhaften Verweis auf eine simperiale Kompatibilität< monumentaler Architektur zu beantworten versucht: ob das Frankreich Ludwigs XIV. oder das Amerika George Washingtons - imperiale Einschüchterungsarchitektur funktioniere in beiden Kontexten im Wesentlichen identisch. Aber Bredekamps wichtige Rehabilitation des Barockgartens sollte Anlass dazu bieten, sich mit oberflächlichen Erklärungen nicht zufrieden zu

\footnotetext{
${ }^{1}$ Eine vorherige Fassung wurde vorgetragen auf der Tagung Politische Ikonographie zwischen produktiver Differenz und Konsens an der Humboldt-Universität Berlin, 3.-4. Dezember 2015. Ich danke den Teilnehmern, insbesondere den Herausgebern, Eva Marlene Hausteiner und Sebastian Huhnholz, sowie Markus Dauss für hilfreiche Kommentare. Teile des Aufsatzes rekurrieren auf Manow 2015.

${ }^{2}$ Bredekamp 2012, S. 78.

3 Ebd., S. 118-119.

${ }^{4}$ Vgl. Sudjic/Jones 2001; Hirst 2005; Sudjic 2006; Field, Gournay et al. 2007; Minkenberg 2014; Parkinson 2014; Benton-Short 2016.

${ }^{5}$ Mumford 1963, S. 469.
} 
geben. Da- rüber hinaus: Die Versailler Vorbildfunktion für die Washington Mall mit Kapitol, Lincoln Memorial, Weißem Haus etc. scheint nicht nur quer zu liegen zu unseren eingeschliffenen Gegenüberstellungen: Barockgarten/Absolutismus versus englischer Landschaftsgarten/liberale Bürgergesellschaft, sondern scheint ebenso wenig zu passen zu einem Frankreich-USA-Vergleich, mit einem zentralistischen Einheitsstaat absolutistischer Provenienz dort und einer föderativen Union demokratischer Einzelstaaten hier. Eignen die sich in einem zentralen Fluchtpunkt treffenden Radialstrahlen gleichermaßen zur Versinnbildlichung des einen wie des anderen, von Einheit wie Vielheit, welcher symbolische Eigenwert würde ihnen dann noch zukommen?

Das verweist aber vielleicht auch nur auf die Grenzen einer Betrachtungsweise, die immer nur nach Materialisierungen von vorgängigen Ideen fragt, und nie nach der nachgängigen Rationalisierung eines bestehenden Inventars von Formen und Materien im Dienste vorgegebener Ideen. $\mathrm{Zu}$ den impliziten Voraussetzungen dieser Betrachtungsweise gehört die Annahme, Ideologien hätten jeweils eine relativ eindeutige Formensprache. Das ignoriert tendenziell die eigenständige politische Prägekraft unserer materiellen Umwelt, und es unterschätzt tendenziell die Flexibilität der Formen und das uneindeutige Verhältnis zwischen symbolischer Form und politischer Norm. Es ist sicherlich eher so, dass wir »mit den Räumen [denken], in denen wir leben $\ll,{ }^{6}$ als dass wir diese Räume direkt denken, also sie nach den Abstrahierungen formen würden, die wir zuvor aus der Anschauung der politischen Wirklichkeit gewonnen hätten. Hat ein demokratischer Souverän in den Raumkategorien der Demokratie gedacht - und das sogar schon um $1790 ?^{7}$ Wohl kaum. Wir haben die Tendenz in den Räumen jeweils das zu sehen, was wir sehen wollen: „under the shadow of Trump - would the classical architecture [of the Lincoln Memorial; PM] suddenly appear squatly fascist? $\ll^{8}(\leftarrow \mathbf{S . ~ 1 8 3})$

Das Bild der Washington-Mall als eines besonders hervorgehobenen Falls von Herrschaftsarchitektur mit ihren Einheits- und Differenzsymbolisierungen soll im Folgenden deswegen nicht als ein Beispiel für die manifest gewordene Absicht verstanden werden, »to create a space representative of American ideals and aspirations $«{ }^{9}$, sondern als Resultat einer Interaktion aus demokratischer Praxis und politischem Raum, einer vielschichtigen Kombination aus Plänen, Design, Gebrauch und Überschreibungen, ein Aneignungsvorgang mit vielfältigen Episoden der Differenz.

\section{Demokratische Aneignung einer barocken Extravaganz}

Als Charles Dickens 1842 Washington D.C. besuchte, sah er »öffentliche Gebäude, die nur eine Öffentlichkeit brauchen, um vollständig zu sein «. ${ }^{10}$ Doch Befürchtungen, L'Enfants barocke Extravaganz würde ein kompletter Fremdkörper in der US-amerikanischen Hauptstadt bleiben, erscheinen heute weitgehend gegenstandslos. Die in den allgemeinen Erinnerungsschatz der Demokratie eingegangenen Bilder des March on Washington for Jobs \& Freedom vom August 1963, die eine Menschenmenge von über 200.000 Protestierenden vor dem Lincoln Memorial und um das reflecting pool herum gelagert zeigen, wie sie der Abschlussrede Martin Luther Kings zuhören, ${ }^{11}$ dokumentieren die exemplarische demokratische Aneignung eines politischen Raums. Zum ersten Mal hatte sich 1894 mit dem Marsch auf Washington von »Coxey's Army« - einer

\footnotetext{
${ }^{6}$ Damler 2012, S. 13.

7 Dunn 2005. Ganz zu schweigen von dem Problem, wie sich das, was um 1800 möglicherweise unter Demokratie verstanden wurde, zu dem verhält, was wir heute unter Demokratie verstehen; vgl. Przeworski 2010.

${ }^{8}$ Amis 2017, S. 19.

${ }^{9}$ Benton-Short 2016, S. 21.

${ }^{10}$ Vgl. Mumford 1963, S. 470.

${ }^{11}$ Siehe etwa https:/www.youtube.com/watch?v=d7s30wFCqlw (Zugriff 17. Mai 2017). Wasserbassins als prominentes Element auch der demokratischen Repräsentationsarchitektur verdienten eine eigene Darstellung; siehe als Auftakt hierzu die Diskussion des Parterre d'Eau von Versailles bei Bredekamp 2012, S. 77-85.
} 
Bewegung von Arbeitslosen, Gelegenheitsarbeitern und Prekarisierten, die in Zeiten der schweren Wirtschaftsdepression von der US-Regierung öffentliche Arbeitsbeschaffungsmaß- nahmen, expansive Geldpolitik und ein schuldenfinanziertes Konjunkturprogramm forderten - diese neue politische Protest- und Artikulationsform etabliert. ${ }^{12}$ Seither hat sie unzählige Wiederholungen erfahren. Was aus der Sicht der einen eine legitime "petition in boots « war, qualifizierte sich aus Sicht der anderen als >Invasion 〈 und unzulässiger Versuch, den formell zuständigen Ort kollektiver Entscheidungsfindung in einer Demokratie, das Parlament, unter den Druck der Straße zu setzen. Als bei der Ankunft in Washington, nach einem über 700 Meilen langen Marsch, der in Ohio seinen Anfang genommen hatte, der Anführer der Bewegung, Jacob Coxey, am 1. Mai 1894 auf den Treppen des Kapitols eine Rede halten wollte, wurde er mit Verweis auf das Verbot politischer Kundgebungen im unmittelbaren Umkreis des Kongresses in Haft genommen. $(\leftarrow \mathbf{S}$. 184)

Die großen Demonstrationen auf Washingtons National Mall bieten uns die Visualisierung eines Transformationsvorgangs, bei dem sich eine unüberschaubare, amorphe Menge, aus dem ganzen Land mit Bussen, Bahnen und Autos zusammenströmend (oder, wie 1894, sich über Wochen zu Fuß - mit kontinuierlicher Medienbegleitung - der Hauptstadt nähernd), nun geometrisch eingefasst von Constitution und Independence Avenue, in der rechteckigen Form der Mall in einen einheitlichen politischen Akteur verwandelt - ein Vorgang, wie sich Vielheit in »ein unauslöschliches Bild der Einheit « ${ }^{13}$ übersetzt, um schließlich den formal zuständigen Ort für die Manifestation des repräsentierten Volkswillens, den US- Amerikanischen Kongress, zu adressieren. Politische Architektur ist hier beides, einerseits statischer Ausdruck: social forces slowed into form und zugleich dynamische Funktion: slowing social forces into form. ${ }^{14}$ Die National Mall mit Lincoln und Washington Memorial, mit dem reflecting pool und der Blickflucht auf den Capitol Hill repräsentiert also mittlerweile eine demokratische Bewegungs- und Transformationsarchitektur im Sinne Ludger Schwartes, der vorschlägt, den politischen Raum eher als »kollektive Interaktion zwischen Menschen und Din- gen [...] zur Festlegung von Aktions- und Passionspotentialen $\ll^{15} \mathrm{zu}$ verstehen, denn als leeres Forum, das zuallererst politisch zu füllen wäre.

Ist es von Bedeutung, dass sich die Menschenmenge im August 1963 nicht nach Westen, zum US-Kapitol, sondern nach Osten, auf das Lincoln Memorial hin ausrichtete? Mit der Regierung war dies abgesprochen, gerade um dem Eindruck eines Versuches der Einschüchterung des Kongresses, in dem zur selben Zeit die Civil Rights-Bill der Kennedy-Administration verhandelt wurde, entgegen zu wirken. Zugleich ist das Lincoln Memorial der Ort der Verewigung jenes konstitutiven Moments in der Geschichte der Vereinigten Staaten, auf dessen Geist sich die Demonstranten beriefen. In den zeitgenössischen Fernsehübertragungen wurde genau dieser Zusammenhang gestiftet: Exakt hundert Jahre nach Lincolns Unterzeichnung der Emancipation Proclamation habe sich eine riesige Menschenmenge vor seiner Statue versammelt, um nun die Rassentrennung endgültig zu überwinden.

Wir sind also auch mit der treffenden architektonischen Versinnbildlichung der dualen Temporalisierung des politischen Systems der Vereinigten Staaten konfrontiert, in dem einer »normalen«, parlamentarischen Politik, manifestiert im US- Kongress (als pouvoir constitué), die singulären Momente »konstitutioneller Politik« gegenüber stehen, in denen die Masse der Bürger (als pouvoir constituant) mobilisiert, um über fundamentale Verfassungsprinzipien zu streiten. ${ }^{16}$ Und diese konstitutionellen Momente der We, the people-Vergewisserung, für Bruce Acker- man im Fall der USA markiert durch die Gründungszeit (Founding Federalists), die Zeit des amerikanischen Bürgerkriegs (Reconstruction Republicans), den New $(\leftarrow \mathbf{S . ~ 1 8 5})$ Deal (New Deal

\footnotetext{
12 Barber 2002.

13 Barber 2002.

14 Weizman 2012.

15 Schwarte 2009, S. 103

${ }^{16}$ Vgl. Ackerman 1993, 1998 und 2014.
} 
Democrats) und nun auch durch das Civil Rights-Movement der 1960er Jahre, finden einerseits ihre besonderen Erinnerungsorte in der Hauptstadtarchitektur, insbesondere in Washington und Lincoln Memorial, sie werden aber andererseits verfassungsrechtlich gepflegt und in der Rechtsprechung des Supreme Court beständig aufgerufen. Das demokratische Spiel zwischen Einheit und Differenz findet innerhalb dieses Ensembles statt: die außergewöhnlichen Momente der Einheitsstiftung schließen die Phasen fundamentalen Streits ab und geben dann den Raum vor, innerhalb dessen das alltägliche Spiel der Differenz stattfinden kann. Die Differenz des täglichen politischen Alltags ist also eingehegt innerhalb eines institutionellen Ensembles, das Ausdruck einer Befriedung grundlegen- der Konflikte ist. An diese konstitutionellen Momente wird durch Monumente erinnert, für Kongress und Oberstes Gericht markieren sie Begrenzungen im Tagesgeschäft des Streits. ${ }^{17}$

Es ist daher ohne große Bedeutung, dass das Gebäude des amerikanischen Verfassungsgerichts nicht direkt in das architektonische Bedeutungsensemble der Mall eingebunden ist, denn einer der zentralen historischen Verfassungsmomente der Republik steht den Repräsentanten im USKongress in der langen Blickflucht der National Mall in Form des Lincoln Memorials ja beständig vor Augen. Bis 1935 aber hatte der Supreme Court tatsächlich direkt im US-Kapitol seinen Sitz. Nach den ursprünglichen Planungen sollte das politische Zusammenwirken von Repräsentantenhaus, Senat und Verfassungsgericht, also die Einheit der Gewaltenteilung, architektonisch im zentralen Dom, der Rotunda des US-Kapitols, versinnbildlicht werden, für die zudem in einer Krypta das Grab George Washingtons vorgesehen war. Die Krypta mit Washingtons republikanischem Heldengrab, da- rüber die anlässlich seines hundertsten Geburtstags 1832 vom U.S. Kongress kommissionierte, umstrittene Marmorstatue, die ihn als Zeus zeigt (und die heute ins National Museum of American History verbannt ist), schließlich im Kuppelfresko der Rotunda die Darstellung der Apotheose Washingtons - dass angesichts $(\leftarrow$ S. 186) dieses völlig überbordenden Symbolprogramms Henry James vom US-Kapitol als baulichem Zeugnis des »bewildered taste« und der »arduous and interrupted education« einer jungen Nation sprach, vermag nicht zu überraschen. ${ }^{18}$

Das verdeutlicht jedoch, wie sehr das, was wir als National Mall kennen, auch die Geschichte der roads not taken, von entweder nicht eingeschlagenen oder sehr bald wieder verlassenen Wegen ist. Die aktuelle Gestalt ist auch das Resultat misslungener Symbolisierungsabsichten, fehlgeschlagener Allegorien, bald als Politkitsch erkannter Formverirrungen, mitunter von »Archiflops «. ${ }^{19}$ So war insbesondere der Rotunda des Kapitols ursprünglich offenbar ein völlig anderer Platz zu- gewiesen worden, sie sollte eigentlich der zentralste Platz des ganzen Ensembles werden. Heute fragen sich viele nur noch: »[W] hy did anyone ever build that huge, expensive round

\footnotetext{
${ }^{17}$ Ein entsprechendes Argument ließe sich für Berlin als neue Hauptstadt machen: Wie die ursprünglich intendierte imperiale Ost/West-Blickflucht von der Siegessäule über das Brandenburger Tor bis hin zum ehemaligen Stadtschloss heute dominiert wird von einer süd-nördlichen Linie aus Holocaust Denkmal, Brandenburger Tor und Reichstag(skuppel), die zudem Albert Speers megalomane Germania-Planungen überschreibt (mit der süd-nördlichen Linie aus Reichskanzlei, Brandenburger Tor und Volkshalle), ist einzigartig dokumentiert in Neil MacGregors Germany - Memories of a Nation, dem Begleitbuch zur gleichnamigen Ausstellung des British Museum; vgl. MacGregor 2014. Siehe insbesondere dort das Foto auf Seite XL-XLI und Kapitel 1 Seeing from a Gate. An diesem Ort mit hohem symbolischen Aussagegehalt stellt sich die Frage nach der Notwendigkeit einer >erinnerungspolitischen<, statt einer parlamentarischen Bannmeile. Nach gängiger Rechtsprechung können Versammlungen in der Nähe von historischen Gedenkstätten verboten werden, wenn sie die Würde der Opfer beeinträchtigen könnten. Siehe hierzu etwa den Entschluss des Bundesverfassungsgerichts bezüglich der Beschwerde der Jugendorganisation der NPD, der Jungen Nationaldemokraten, gegen die Untersagung der Route einer für den 8. Mai 2005 geplanten Demonstration (vorbei am Holocaust-Denkmal und Brandenburger Tor) durch den Berliner Polizeipräsidenten (Beschluss vom 06. Mai 2005 - 1 BvR 961/05).

${ }^{18}$ Zitiert nach Scott 1995, S. 8. Der Plan für Washingtons Grab im Dome des US-Kapitols wurde erst aus Kostengründen, dann wegen des Widerstands der Familie gegen eine Umbettung, nicht verwirklicht.

${ }^{19}$ Biamonti 2017.
} 
room in the middle of the Capitol, that has never, to the present minute, served any coherent and convincing purpose? $\ll^{20}$

In den ursprünglichen Planungen war die Rotunda ganz im Gegenteil völlig überladen mit symbolischem Gehalt, sie sollte ein zivilreligiöser Tempel sein als freie Variation des römischen Pantheons: »a Roman temple slid into the center of a larger building « ${ }^{21}$ Die Pläne Thorntons, des Architekten des Kapitols, sahen für die Rotunda eine umfangreiche Marmorgruppe vor, für deren Herstellung eine Reihe europäischer Bildhauer nach Washington gebracht werden sollten. Thornton stellte sich »an elaborate group in white marble « vor, einen großer Fels aus weißem Marmor in der »Bernini-Falconet Tradition«, in ihm das Grab Washingtons, und auf dem Fels eine Reihe allegorischer Figuren. Insbesondere: »A female figure symbolizing Eternity would lead the figure of Washington to the peak of the rock, to take flight into the heavens «. ${ }^{22}$ Das ist uns heute schwer erträglicher Politkitsch, der dann im Fresko der Kuppel mit seiner Apotheose-Szene wieder aufgenommen worden wäre.

Anhand der National Mall ließe sich also exemplarisch zeigen, dass der öffentliche Raum immer grundsätzlich umstritten bleibt und dass seine Wirkung nie in den Absichten seiner Gestalter vollständig aufgeht. Das ließe sich schon an den Dimensionen der ursprünglichen Planungen ersehen, die sich für Jefferson nur als »lavish «, kryptomonarchisch und als Ausdruck einer unguten "grandomania« darstellten. ${ }^{23}$ Die beabsichtigte Grandeur wird schon an der Grundfläche der ursprünglichen Planungen offensichtlich. L'Enfants Stadtplan umfasste »five $(\leftarrow \mathbf{S}$. 187) thousand acres, comparable at the time to the footprints of Paris and London and as large as New York City, Boston, and Philadelphia combined ${ }^{24}$

Das verdeutlicht recht gut das leicht Megalomane des Vorhabens, insbesondere wenn man sich veranschaulicht, in welchem erbärmlichen Zustand sich die Nation um 1790 befand: »fiskalisch ruiniert durch den Revolutionskrieg, geführt durch einen Kongress, der einer Zentralisierung der Macht hochgradig ambivalent gegenüber stand, und bevölkert durch Bürger, die über ein riesiges Land verstreut waren und wenig reisten, so dass sie weder viele Gründe noch viele Gelegenheiten hatten, so etwas wie ein Nationalbewusstsein zu entwickeln ${ }^{25}$. Die Absicht, ein Einheitssymbol für die neue Nation zu schaffen, mag handlungsleitend gewesen sein, aber man fragt sich, an wen genau sich das ursprünglich eigentlich richten sollte.

Die räumlichen Zwecksetzungen sind in also ihrer Schaffung kontrovers und ihrer Wirkung ambivalent und gewinnen erst durch eine Öffentlichkeit, die sich im politischen Raum verhält, ihren besonderen und daher auch über Zeit veränderlichen Sinn. Das ist, so möchte man meinen, zumindest das Spezifische an der politischen Architektur in der Demokratie, dass sie nie ohne eine völlig autonome Reaktion ihres Adressaten, des souveränen Volks, entstehen kann (und Teil seiner unvorhersehbaren Reaktion die Umnutzung vordemokratischer Architektur ist). Und dass überhaupt das souveräne Volk zugleich Schöpfer und Adressat dieser Architektur ist, sie also nur als eine Form der kollektiven Selbsteinwirkung verstanden werden kann. Wie alle Herrschaftsarchitektur wollen auch die Bauten der Demokratie Souveränität verkörpern, aber im demokratischen Kontext ist der Souverän »a rather abstract and broadly collective one: the people « ${ }^{26}$ Und je mehr dieser Souverän am Entscheidungsprozess über die konkrete Form seiner eigenen politischen Repräsentation beteiligt ist, desto weniger ist mit einer eindeutigen, spezifischen Formsprache zu rechnen, wie Michael Minkenberg argumentiert. Denn warum sollte sich der demokratische Souverän binden?

\footnotetext{
${ }^{20}$ Green 2002.

21 Ebd.

22 Green 2002.

23 Parkinson 2014, S. 71.

${ }^{24}$ Benton-Short 2016, S. 24-25.

${ }^{25}$ Luria 2005, S. 155; meine Übersetzung.

${ }^{26}$ Minkenberg 2014, S. 53.
} 


\section{Die Mall als zeremonieller Ort - Einheit und Differenz des Souveräns}

Die seit 1981 an der Westfront des Kapitols, also der dem Lincoln Memorial zu- gewandten Seite stattfindenden Inauguration des US-Präsidenten mit Vereidigung und Antrittsrede verwandelt die National Mall in einen »zeremoniellen Raum «, ${ }^{27}$ die Menge ist nun Publikum, nicht Akteur. $\mathrm{Zu}$ Obamas Vereidigung als 44. Präsidenten der USA am 20. Januar 2009 kamen 1,8 Millionen Menschen - die größte jemals auf der Mall versammelte Menschenmenge, sodass sie zum ersten Mal in $(\leftarrow \mathbf{S}$. 188) ihrer vollen Länge freigegeben wurde. Die zum US-Kapitol hinaufführenden Treppen werden für die Zeremonie zu einer Art Amphitheater umgebaut, das nun jedoch hinter dem Rednerpult, im Rücken des Redners in Richtung der Mall geöffnet ist. Im Hintergrund ragt der Kongress mit dem zentralen Dom über hundert Meter imposant in die Höhe. Auf den Rängen sitzen offizielle Gäste, Familienfreunde und Prominenz.

Der Ort von Obamas Inauguration ermöglichte eine Politik der kleinen Zeichen, um den Zusammenhang mit der Civil Rights-Bewegung, die fast fünfzig Jahre zuvor auf der National Mall ihre berühmteste Manifestation gefunden hatte, herzustellen: Mit John Lewis befand sich der einzige noch lebende Redner des 1963er Marsches auf Washington unter den geladenen Gästen, Obamas Antritts- rede zitierte mit dem Motto >A New Birth of Freedom< Lincolns Gettysburg Address und verwies auf das sich zum zweihundertsten Male jährende Geburtsjahr des 16. USPräsidenten. ${ }^{28}$ Warum aber wird der Präsident auf den Stufen des US- Kapitols, dessen Kammern er nur auf besondere Einladung betreten darf, und nicht am oder im Weißen Haus vereidigt? Erst nach der Inauguration mit Ablegen des Amtseides wird der Präsident mit einer Eskorte die Pennsylvania-Avenue hinunter zum Weißen Haus geführt.

In der repräsentativen Demokratie stehen Parlamente und die versammelte politische Menge in einem besonderen Verhältnis zueinander, die - was ihre symbolische Darstellung angeht vielleicht am treffendsten in einem Urteil zur Bannmeile des Kapitols als Zielkonflikt zwischen der "glorification of a form of government through visual enhancement of its public buildings « auf der einen Seite und dem bürgerlichen »exercise of [...] basic constitutional rights in their most pristine and classic form « auf der anderen beschrieben wurde. ${ }^{29}$ Es ist die Konkurrenz zwischen der auf Dauer gestellten, d.h. auch baulich verfestigten formalen Repräsentation und der spontanen politischen Versammlung. Das Feste und das Flüssige, l'espace strié und l'espace lissé, politische Architektur als »social forces slowing into form $«{ }^{30}$ die aber etwas von dem Flüssigen dieser gesellschaftlichen Kräfte noch referenziert: vielleicht rührt hiervon die Vorliebe, massive politische Repräsentationsbauten sich in vorgelagerten Wasserflächen spiegeln zu lassen?

In der Inaugurationszeremonie ist dieser Souveränitätstransfer selbst noch ein- mal inszeniert, denn die grundlegende Konstellation ist ja eine zwischen versammelter Menge und neuem Amtsinhaber. Zeremonie und Demonstration, wie bei Trump die Inauguration und direkt am darauf folgenden Tag der Women's march on Washington, stellen zwei unterschiedliche Arten von politischen Mengen dar. So wird es in der Demokratie zur Aufgabe, die Frage zu klären - und sie auch räumlich zu klären: was ist eine Menge? Und was macht eine Menge zur politischen $(\leftarrow$ S. 189) Menge? Und wie ist das Verhältnis der fluiden Menge zu den institutionalisierten, verfestigten Formen ihrer politischen Repräsentation?

Fragen dieser Art werden juristisch einerseits im Versammlungsrecht, spezifischer in der Einrichtung von Bannmeilen geregelt, die nun den liminal space zwischen Beherrschten und Herrschenden markieren, ${ }^{31}$ andererseits aber auch im Parlamentsrecht, das aus der

27 Vgl. Goodsell 1988, S. 12. Von Washington bis Carter, gut zweihundert Jahre lang, wurden die Inaugurierungsfeierlichkeiten am Ostportikus des Kapitols abgehalten, erst Reagan brach mit dieser Tradition.

${ }^{28}$ Es ist genau jener John Lewis, der erklärte, er werde der Inauguration Trumps fernblei- ben, weil er ihn nicht als legitimen Präsidenten anerkenne, was Trump mit einer Reihe aggressiver Tweets beantwortete.

29 Jeannette Rankin Brigade v. Chief of Capitol Police (D.D.C. 1972). See, for instance: http://law.justia.com/cases/federal/district-courts/FSupp/342/575/2339637/.

${ }^{30}$ Weizman 2012.

${ }^{31}$ Breitbach 1994. 
Binnenperspektive den Zugang zum Parlament, Petitionsrechte und die Öffentlichkeit der parlamentarischen Verhandlungen, also von Innen das parlamentarische Innen/ Außen reguliert bis hin zum Verbot der Äußerung von Zustimmung oder Ablehnung auf der Zuschauergalerie im Parlament. In den USA verbot der Act to Regulate the Use of the Capitol Grounds von 1882 politische Demonstrationen in der Nähe des Kongresses. Diese Regel wurde erst Anfang der 1970er Jahre im Zuge des Anti-Vietnam-Protestes abgeschafft - vorherige Demonstrationen beruhten auf Ausnahmegenehmigungen im Einzelfall. ${ }^{32}$

Aber was macht nun eine Menge zu einer politischen Menge? Ohne Zweifel ist die schlichte Größe ein zentrales Kriterium. Auch die Organisatoren des Marsches auf Washington von 1963 verwiesen auf die schlichte significance of our numbers - in der Demokratie grundsätzlich ein gewichtiges Argument, denn die versammelte Menge bietet einen Vorschein auf die wählenden Bürger. ${ }^{33}$ Auch in dieser Hinsicht kommunizieren der informelle und der formelle Souverän in der Demokratie. Die Obama-Inauguration zeigt, so John Parkinson, »that sometimes very, very large number of people think that $[\ldots]$ the physical occupation of space $[\ldots]$ does matter. $\ll^{34}$

Der bizarre Streit um die Zahl der Zuschauer, die sich zu Trumps Vereidigung am 20. Januar 2017 auf der Mall versammelt hatten, veranschaulicht das noch einmal in allen Details. Der Streit der Bilder und der Zahlen drehte sich um eine für Demokratien zentrale Frage. Wie zählt man politisch, wie zählt man eine Menge ${ }^{35}$ Ein gängiges Verfahren, das auch bei der National Mall lange zur Anwendung kam, ist das der Luftbilder, denen ein GPS-Grid unterlegt wird, wodurch (૯ S. 190) dann unter der Annahme eines durchschnittlichen Personenabstands (eine locker zusammenstehende Menge: fast ein Quadratmeter pro Person; eine dicht gedrängte Menge: etwas mehr als ein halben Quadratmeter pro Person) die Gesamtzahl der Teilnehmenden hochgerechnet werden kann. Aber Luftbilder bei Großereignissen sind in Washington schlecht zu machen, denn einerseits gibt es im Umfeld der Mall mit ihren großen Abmessungen keine hohen Häuser, andererseits erlauben die strikten Sicherheitsvorkehrungen keine Drohnen oder den Überflug der Veranstaltung mit Flugzeugen oder Hubschraubern.

Die nationale Parkverwaltung als für die National Mall verantwortliche Behörde hatte bis 1996 Luftbilder zur Abschätzung der Größe von Mengen verwendet. Doch beim Million Man March on Washington von 1995 waren die offiziellen Zahlen zum Gegenstand heftigen Streits geworden und danach entschloss sich die Parkverwaltung, auf die Schätzung und Bekanntgabe von Teilnehmerzahlen künftig zu verzichten. Während der National Park Service die Teilnehmerzahl des Million Man March mit 400.000 angegeben hatte, meinten die Veranstalter, ihr Eine- MillionZiel sei erreicht worden. Nation of Islam-Führer Louis Farrakhan, einer der Organisatoren des Marsches, drohte der Parkverwaltung mit Klage und sah die niedrigen Schätzzahlen als Beweis dafür, dass in der Behörde »racism, white supremacy and hatred for Louis Farrakhan« vorherrschen würden. ${ }^{36}$ Wissenschaftler von der Boston University schätzten in einer unabhängigen Untersuchung die Teilnehmerzahlen tatsächlich deutlich höher, mehr als doppelt so hoch wie von

\footnotetext{
${ }^{32} \mathrm{Im}$ britischen Fall datiert ein Gesetz, das politische Demonstrationen im Umkreis von einem Kilometer vom Parlament verbietet, erst aus dem Jahr 2005. Auf einen Dauerprotestler, Brian Haw, der seit Juni 2001 vor dem Big Ben kampierte um auf das Schicksal irakischer Kinder aufmerksam zu machen, konnte es keine Anwendung finden. Auch das Preußische Vereinsgesetz vom 11. März 1850 bestimmte in seinem $\S 11$, dass >innerhalb zweier Meilen von dem Ort der jeweiligen Residenz des Königs oder der beiden Parlamentskammern Volksversammlungen nicht gestattetく seien. »Dieser Vorschrift liegt die Erwägung zugrunde, daß von den Entschließungen der höchsten Staatsgewalten selbst der Schein ferngehalten werden muß, als könnten sie unter dem Einfluß von Versammlungen gefaßt worden sein, welche sich als Vertreter des Volks- willens aufwerfen möchten « - so wird es in einem Kommentar zur Bannmeilenregelung im Preußischen Verwaltungsblatt ausgeführt; vgl. Delius 1907. Aber wann ist eine Menschenmenge eine Volksversammlung?

${ }_{33}^{33}$ Mansbridge 2003.

${ }^{34}$ Parkinson 2014, S. 99.

${ }^{35}$ Schwartzberg 2014.

${ }^{36}$ Siehe http://bigstory.ap.org/article/7afad98b7d78423cbb5140fe810e3480/when-it-com es-inaugural-crowdsdoes-size-matter (Zugriff 17. Mai 2017).
} 
der Parkverwaltung angegeben. Ab 1996 schließlich berichtete der Park Service wegen der regelmäßigen Kontroversen keine Teilnehmerzahlen mehr.

Wenn man keine offiziellen Zahlen bekommt, können die Zahl verkaufter Metro-Tickets in den Stunden vor dem Ereignis über die Teilnehmerzahlen Aufschluss geben, aber natürlich ist das ebenfalls kein präzises Verfahren, sondern ergibt höchstens Näherungs- und Vergleichswerte. WMATA, die Washington Metropolitan Area Transit Authority, berichtete für den Tag von Trumps Inauguration von 193.000 verkauften Tickets bis um 11 Uhr des Tages. Zur selben Zeit 2009, bei Obamas' erstem Amtseid, waren es laut WMATA 513.000 verkaufte Tickets gewesen, zu seiner zweiten Amtszeit 317.000, für Bush jr. zweite Amtszeit 197.000 Metrofahrer bis $11 \mathrm{Uhr}^{37}$ Wo die Zahlen nicht eindeutig sind, kann der Streit als Vergleich der Bilder ausgetragen werden, und so ging die direkte Gegenüberstellung der Luftaufnahmen von Trumps Inauguration (Abb. 1) und von der Obamas (Abb. 2) um die Welt. Aber jedes Bild hat als politisches Argument nur $(\leftarrow$ S. 191) begrenzte und für die jeweiligen Lager jeweils ganz andere Aussagekraft, und neben dem alternativen Fakten gibt es immer auch alternative Bilder. Entscheidend für die Trump Anhänger war dann tatsächlich ein ganz anderer Größenvergleich (Abb. 3).

Abb. 1 und 2: Vergleich der Teilnehmergröße der Inaugurationsfeierlichkeiten am 20. Januar 2017

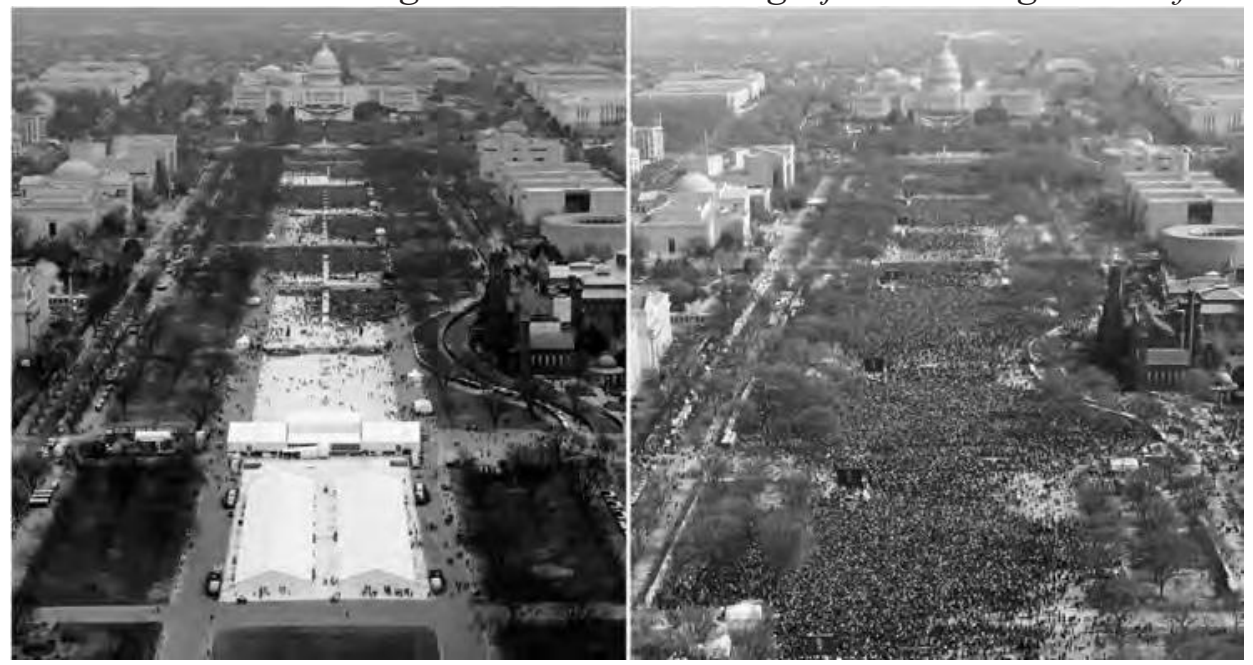

(Trump; links) und am 20. Januar 2009 (Obama, rechts).

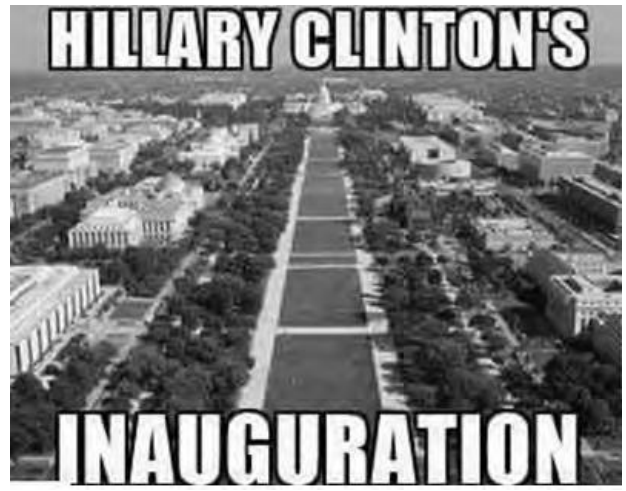

Abb. 3: Meme zum Streit um den Vergleich der Teilnehmerzahlen zwischen Obamas und Trumps Inauguration.

\footnotetext{
37 Schätzungen der Teilnehmerzahlen bei den Vereidigungszeremonien in Washington D.C.: Bill Clinton (1993, erster Term): 800.000 Zuschauer, Clinton (1997, zweiter Term): 250.000 Zuschauer, George W. Bush (2001, erster Term): 300.000 Zuschauer, George W. Bush (2005, zweiter Term): 400.000 Zuschauer; Barack Obama (2009, erster Term): 1,8 Millionen Zuschauer, Barack Obama (2013, zweiter Term): eine Million Zuschauer; die geschätzten 700.000 bis 900.000 Besucher bei Trump (2017) wären im Vergleich immer noch eine recht große Menge.
} 
Am 21. Januar, einen Tag nach der Vereidigung Donald Trumps, kamen zum Women's March on Washington eine um die 500.000 geschätzte Zahl von Teilnehmern und Teilnehmerinnen, diesmal veröffentlichte die WMATA die Zahl von 273.000 verkauften Metro-Tickets bis $11 \mathrm{Uhr}$, acht Mal soviel wie an einem normalen Samstag um diese Uhrzeit und deutlich höher als die Zahl vom Vortag. $(\leftarrow$ S. 192)

Landesweit wird die Zahl der Teilnehmerinnen auf drei Millionen geschätzt. Das wäre die größte Demonstration in der US-amerikanischen Geschichte.

Der Zahlenstreit ist konstitutiv für die Demokratie, ${ }^{38}$ und die Kontroverse über die crowd size bei Trumps Vereidigung zeigt, dass die Zahl in der Wahl besser zu ermitteln ist als in der Versammlung. ${ }^{39}$ Vor jeder Frage nach einer substantiellen Beteiligung an der Formulierung des Kollektivwillens: kein »demokratischer Wille [...] ohne Regeln, die bestimmen, wie dieser Wille entstehen soll. [...] Ein demokratischer Wille besteht [...] niemals allein aus einer formlosen Zusammensetzung individueller Beiträge wie bei einer Demonstration [...], weil [...] nicht sichergestellt ist, dass alle die freie und gleiche Möglichkeit der Mitentscheidung haben ${ }^{40}$ - also bereits vor Klärung dieser inhaltlichen Frage geht es in der Demokratie zu- nächst um die Klärung der rein quantitativen Dimension: Wie viele? Aufgrund von Repräsentation lasse sich »diese aus potentieller Macht sich bildende aktuale Macht quantitativ abmessen; in diesem Sinne wird Macht überhaupt erst berechenbar. $\aleph^{41}$ Und dabei geben Wahlen weniger Gelegenheit für alternative Fakten:

»This was the largest audience to ever witness an Inauguration, period, both in person and around the globe« (Pressesprecher des Weißen Hauses, Sean Spicer, am 21. Januar 2017). Es ist daher die präzise gezählte und dann repräsentierte Menge die zählt in der Demokratie, in Differenz zur bloß versammelten. ${ }^{42}$ Im Rahmen der revolutionär neuen Form von Politik, für die Trump prototypisch steht, ist es aber dann vielleicht auch eher die virtuell versammelte Menge der Follower (wieviele davon sind fake accounts?), die seinen Tweets folgt, die politisch als Menge maßgeblich wird. Gleichwohl wird uns die Einheit der Differenz zwischen Repräsentierten und dem Repräsentanten, zwischen der Menge und dem Präsidenten, in der alle vier Jahr stattfindenden Inaugurationszeremonie auf der National-Mall immer wieder vorgeführt.

\section{Political time, slowing into form}

Wie kanalisiert und/oder domestiziert die vorgegebene Architektur in ihrem weitesten Sinne die je konkreten und gesellschaftlich dynamischen Ansprüche und Massenartikulationen? Diese Frage wurde hier anhand der National Mall in Washington D.C. gestellt. Die Mall ist Ort der Zeremonie, der rituellen Versammlung $(\leftarrow \mathbf{S}$. 193) des Volkes und seiner im Vergleich dazu mehr spontanen Versammlung, also der Demonstration. Sie ist damit Ort der Einheit der Differenz aus Repräsentierten und Repräsentanten. Und im kollektiven Imaginären, als manifest-gewordene Konstituierung der politischen Gemeinschaft, ist die Mall auch immer wieder Phantasieort der Destruktion gewesen, der vorgestellten völligen Zerstörung (und Wiedererrichtung): Independence Day (1996), White House Down (2013), Olympus Has fallen (2013). Damit hat die Mall aber nicht nur die Fantasie der Filmemacher beschäftigt. Der United-Airlines-Flug 93, eines der vier

\footnotetext{
38 Schwartzberg 2014.

${ }^{39} \mathrm{Vgl}$. Manow 2017.

${ }^{40}$ Möllers 2008, S. 28.

41 Vollrath 1993, S. 67.

$42 »$ Das Volk ist eine Einheit (unum quid) mit einem Willen und ist einer Handlung fähig; all das kann von einer Menge nicht gesagt werden. [...] Gemeine Leute und andere, die den Sachverhalt nicht erfassen, sprechen von einer großen Zahl Menschen immer als vom Volke. [...]. Allein sie wiegeln dabei unter dem Vorwand, dass es das Volk sei, die Bürger gegen den Staat, d. h. die Menge gegen das Volk auf« (Hobbes 1994 [1641], S. 198f. [12/8]).
} 
Flugzeuge der koordinierten Terrorattacke vom 11. September 2001, der über Pennsylvania aufgrund einer Passagierrevolte abstürzte, sollte aller Wahrscheinlichkeit nach in das Weiße Haus oder alternativ das Kapitol gesteuert werden. Die ultimative Form politischer Einheit wird in den Kategorien von Freund und Feind generiert, in An- griff auf und Verteidigung gegen, und das Gemeinwesen konstituiert sich dann als Gegner oder gegen den Gegner.

\section{Literaturverzeichnis}

Ackerman, Bruce A. 1993. We The People: Volume 1 Foundations. Cambridge, Mass.: Belknap Press.

Ders. 1998. We the People: Volume 2, Transformations. Cambridge, Mass.: Harvard UP.

Ackerman, Bruce A. 2014. We the People: Volume 3 The Civil Rights Revolution. Cambridge, Mass.: Harvard UP.

Amis, Louis 2017. «Donald Trump’s Inauguration«, in Times Literary Supplement, No. 5939, S. 19.

Barber, Lucy 2002. Marching on Washington: the forging of an American political tradition. Berkeley, CA: California UP

Benton-Short, Lisa 2016. The National Mall: No Ordinary Public Space. Toronto: Toronto UP Biamonti, Allessandro 2017. Archiflop: Gescheiterte Visionen. Die spektakulärsten Ruinen der modernen Architektur. München: Deutsche Verlagsanstalt.

Bredekamp, Horst 2012. Leibniz und die Revolution der Gartenkunst. Herrenhausen, Versailles und die Philosophie der Blätter. Berlin: Wagenbach.

Breitbach, Michael 1994. Die Bannmeile als Ort von Versammlungen: Gesetzgebungsgeschichte, verfassungsrechtliche Voraussetzungen und ihre verfahrensund materiellrechtlichen Folgen. Baden-Baden: Nomos.

Damler, Daniel 2012. Der Staat der Klassischen Moderne. Berlin: Duncker \& Humblot.

Delius 1907. „Das Verbot von Volksversammlungen am Aufenthaltsorte des Kaisers und Königs sowie am Sitz der Parlamente«, in Preußisches Verwaltungsblatt 28, 14, S. 253-255.

Dunn, John 2005. Democracy - a History. New York: Atlantic Monthly Press.

Field, Cynthia. R. et al. 2007. Paris on the Potomac: The French Influence on the Architecture and Art of Washington, D.C. Ohio: Ohio UP.

Goodsell, Charles T. 1988. The Social Meaning of Civic Space. Studying political Authority through Architecture. Kansas: Kansas UP.

Green, Bryan C. 2002. »George Washington Equestrian Statue« (Entwurf), http://www.vacapit ol.org/documents/NR_Richmond_GeorgeWashingtonEquestrianStatue_127-6084_text.pdf (Zugriff 22. Juli 2017).

Hirst, Paul Q. 2005. Space and power: politics, war and architecture. Cambridge: Polity. Hobbes, Thomas 1994 [1642]. De Cive (Vom Menschen - Vom Bürger: Elemente der Philoso-

phie II und III, hrsg. v. Gawlick, Günter). Hamburg: F. Meiner.

Luria, Sarah 2005. Capital Speculations: Writing and Building Washington, D.C. New Hampshire: New Hampshire UP.

MacGregor, Neil 2014. Germany-Memories of a Nation. London: Allen Lane. $(\leftarrow$ S. 195)

Manow, Philip 2015. »Demokratie und Architektur«, in Merkur. Deutsche Zeitschrift für Europäisches Denken 69, 3, S. 45-52.

Ders. 2017. Die zentralen Nebensächlichkeiten der Demokratie. Von Applausminuten, Föhnfrisuren und Zehnpunkteplänen. Reinbek bei Hamburg: Rowohlt (Polaris).

Mansbridge, Jane 2003. »Rethinking Representation«, in American Political Science Review 97, 4, S. 515-528.

Minkenberg, Michael 2014. » A City of the People, by the People, for the People. Democracy and Capital-Building in Washington, DC, Ottawa, Canberra, and Brasilia«, in Power and Architecture. The Construction of Capitals and the Politics of Space, hrsg. v. Minkenberg, 
Michael, S. 53-105. New York: Berghahn.

Ders. (Hrsg.) 2014. Power and Architecture. New York: Berghahn Books.

Möllers, Christoph 2008. Demokratie - Zumutungen und Versprechen. Berlin: Wagenbach.

Mumford, Lewis 1963. Die Stadt. Geschichte und Ausblick. Köln: Kiepenheuer \& Witsch.

Parkinson, John 2014. Democracy and Public Space: The Physical Sites Of Democratic Performance. Oxford: Oxford UP.

Przeworski, Adam 2010. Democracy and the Limits of Self-Government. New York: Cambridge UP.

Schwarte, Ludger 2009. Theorie der Architektur. München: Fink.

Schwartzberg, Melissa 2014. Counting the Many. The Origins and Limits of Supermajority Rule. New York: Cambridge UP.

Scott, Pamela 1995. Temple of Liberty. Building the Capitol for a New Nation. Oxford: Oxford UP.

Sudjic, Deyan 2006. Der Architekturkomplex. Monumente der Macht. Düsseldorf: Artemis \& Winkler.

Sudjic, Deyan; Jones, Helen 2001. Architecture and Democracy. London: Laurence King Publ.

Vollrath, Ernst 1993. »Identitätsrepräsentation und Differenzrepräsentation«, in Recht und Moral, hrsg. v. Orsi, Giuseppe et al., S- 65-78. Frankfurt a. M. u.a. Peter Lang.

Weizman, Eyal 2012. "Agents and Sensors«, in What is Critical Spatial Practice?, hrsg. v. Hirsch, Nikolaus; Miessen, Markus, S. 143-145. Berlin: Sternberg Press.

\section{Abbildungsverzeichnis}

Abb. 1: https://www.cbsnews.com/news/photos-president-trumps-inauguration-crowd-vspresident-obamas/ (Zugriff vom 26. September 2017), (C) Reuters.

Abb. 2: https:/www.cbsnews.com/news/photos-president-trumps-inauguration-crowd-vspresident-obamas/ (Zugriff vom 26. September 2017), (C) Reuters.

Abb. 3: http:/starecat.com/hillary-clintons-inauguration-no-people-empty-field/ (Zugriff vom 26. September 2017). ( $\leftarrow$ S. 195) 